\title{
Study of multiple antennas with defected ground slot for low-band LTE application
}

\author{
Wai Loon Cheor, Azremi Abdullah Al-Hadi, Ping Jack Soh, Mohd Faizal Jamlos \\ Advanced Communication Engineering (ACE) Center of Excellence, \\ School of Computer and Communication Engineering, Universiti Malaysia Perlis, Malaysia
}

\begin{abstract}
Article Info
Article history:

Received Nov 4, 2018

Revised Dec 8, 2018

Accepted Jan 2, 2019

\section{Keywords:}

Defected ground slot

Low frequency

Multiple antennas

Mutual coupling

ABSTRACT

This study is focused on highly coupled multiple antennas with defected ground slot techniques. Two Printed Inverted-F Antenna (PIFA) are positioned at the top edge of chassis symmetrically. Both antennas are operating at low-band Long-Term Evolution (LTE) with center frequency, $829 \mathrm{MHz}$. Rectangular defected ground slot is implemented to reduce the coupling effect between the antennas on the ground plane of the small chassis. Parameter study of the rectangular defected ground slot is studied with different width, $\mathrm{W}$ and length, L. Furthermore, the optimized dimensions of rectangular defected ground slot, $\mathrm{W}$ and $\mathrm{L}$ are simulated and presented. The optimized defected ground slot reduced the mutual coupling up to $-4.5 \mathrm{~dB}$. The envelope correlation coefficient (ECC) achieved less than 0.5 . The ground plane of the multiple antenna structure has been further investigated by introducing another slot with a gap of $1 \mathrm{~mm}$ between them. The achieved result is not significant in term of S-parameter and ECC compared to single defected ground slot.
\end{abstract}

Copyright (C) 2019 Institute of Advanced Engineering and Science. All rights reserved.

\section{Corresponding Author:}

Wai Loon Cheor,

Advanced Communication Engineering (ACE) Center of Excellence,

School of Computer and Communication Engineering,

Universiti Malaysia Perlis, 01000 Kangar, Perlis, Malaysia.

Email: wlcheor@gmail.com

\section{INTRODUCTION}

In recent decades, there is an increasing demand in higher data rate transmission specifically in the development of wireless communication. Multiple Input Multiple Output (MIMO) is a promising technology to improve the channel capacity with multiple antennas. MIMO technology is widely used in mobile terminal to improve the device data rate performance. However, the increasing number of antennas creates a high mutual coupling. Mutual coupling between antennas greatly degrade the overall operating performance [1]. However, the mutual coupling is usually compensated with a distance between antennas by a quarter wavelength [2]. Due to the limited size of terminal chassis in mobile terminals, it is challenging to locate multiple antenna on the small chassis with a promising performance [3]. In other words, chassis with limited space on mobile terminal is challenging to locate multiple antenna with a distance less than a quarter wavelength. The aforementioned problem is significant when the operating frequency is less than $1 \mathrm{GHz}$.

There are several techniques to compensate the mutual coupling for multiple antennas, i.e. defected ground plane [4-11], parasitic elements [12, 13] and decoupling network [14, 15]. Mutual coupling had been reduced by implementing with these techniques but there is limitation. This paper is focusing on decoupling technique of defected ground plane. Several studies have implemented defected ground plane to reduce the mutual coupling among antennas [4-8]. The operating frequency in [4, 5-7] and [8] are $1.8 \mathrm{GHz}, 2.5 \mathrm{GHz}$ and $>3 \mathrm{GHz}$, respectively. Some authors implemented more than one technique to reduce the mutual coupling [9-11]. In [9], an addition connecting line is added between antennas with defected ground plane to enhance 
the impedance matching and mutual coupling at 1.71-2.72 GHz. In [10], parasitic element and defected ground plane are implemented to reduce the coupling at $2 \mathrm{GHz}$. Apart from the defected ground slot, lumped elements were used to improve the inductance of the slot to decouple at frequency below $1 \mathrm{GHz}$ in [11]. Most of these papers focused on decoupling at high frequency.

This paper investigates the effect of defected ground structure with PIFA for the low-band LTE (698-960 MHz) in a small mobile terminal. The center frequency, $\mathrm{f}_{\mathrm{c}}$ of the antenna is $829 \mathrm{MHz}$. The effect with the present of defected ground in different dimension and its performance is studied. Other than Sparameters, the performance, such as total efficiency and ECC are presented. This is to provide the insight concerning impact of defected ground plane on mutual coupling and ECC below $1 \mathrm{GHz}$. Section II presents the design of the antenna structure and the parameters of defected ground structure varied in this study. The characteristic of parameters varied are discussed with performance metric, i.e. mutual coupling and total efficiency. The optimized results are presented and discussed in Section III, and conclusions are revealed in Section IV.

\section{PARAMETER STUDY ON DEFECTED GROUND PLANE}

\subsection{Antenna structure}

This paper utilized the meander PIFA structure in [16]. Dimensions of antenna structure are shown in Figure 1. The dimensions of chassis used are $110 \times 55 \times 1.52 \mathrm{~mm}^{3}$ with Rogers substrate. The overall dimensions of the meander PIFA are $23 \mathrm{~mm}$ x $20 \mathrm{~mm}$. Antenna element 1 (A1) and antenna element 2 (A2) are positioned symmetrically at the top edge of the chassis. The gap between A1 and A2 is $0.042 \lambda$. The studied defected ground plane is a rectangular slot with $\mathrm{W} \times \mathrm{L} \mathrm{mm}$ in between of A1 and A2.

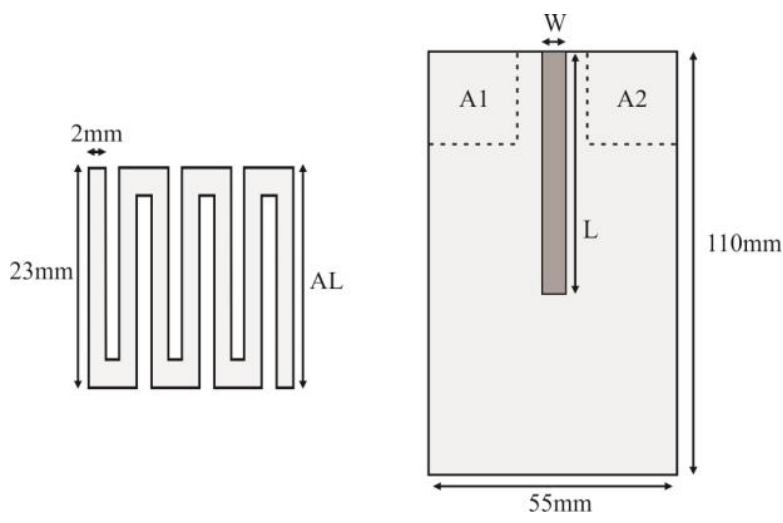

Figure 1. Geometry of antenna structure

\subsection{Characteristic of performance on parameter study}

In this study, the current distribution of the ground plane is monitored without the present of defected ground slot. This is to determine the best location to locate the defected ground slot. Based on the observation, high current distribution is found between the antennas on the ground plane. This location gives highest coupling effect. Hence, the location of the defected ground slot is positioned at the top edge of the chassis as shown in Figure 1.

Characteristic of the defected ground slot is observed by varying the dimensions of the slot. The first part of this study focused in the variation in length of the slot, $\mathrm{L}$ where $\mathrm{W}$ is fixed at $0.0042 \lambda$. The dimension $\mathrm{L}$ is varied from $0.05 \lambda$ to $0.3 \lambda$. Figure 2 shows the effect of varying $\mathrm{L}$ on the ground plane on $\mathrm{S}$-parameters. The A1 and A2 are positioned symmetrically, hence, $S_{11=} S_{22}$ and $S_{12}=S_{21}$. The increment in the dimension $L$ caused the $\mathrm{f}_{\mathrm{c}}$ shifted.

Figure 3 shows the relation between varied $L$ and the studied performance. The $f_{c}$ is given a negative sign (-) when shifted to a lower frequency and vice versa. In Figure 3(a), the S-parameters (decibel, dB) and the frequency detuned $(\mathrm{MHz})$ are denoted by the left and right of the $y$-axis, respectively. The varied length is implied in lambda at the X-axis. The frequency is detuned to a lower frequency at two different points when the $\mathrm{L}$ is increasing. The first point is started from the origin and the second point is started at $900 \mathrm{MHz}$.

The initial value of the mutual coupling is $-2.8 \mathrm{~dB}$. It is possible to have a small reduction on mutual coupling along the variation of $\mathrm{L}$. Mutual coupling is maintained at $-6 \mathrm{~dB}$ started from $0.15 \lambda$ to $0.25 \lambda$. However, the impedance matching is low at $0.15 \lambda$ as compared to others length. Based on Figure 3 (a), 0.25 
$\lambda$ is found to have a good impedance matching and a lower mutual coupling. This shows that $\lambda / 4$ can provides a promising performance as compared to other length of $\mathrm{L}$ with the exclusion of frequency detuned. Figure 3(b) shows the total efficiency and envelope correlation coefficient (ECC) for parameter sweep, L. The result is obtained by selecting several operating frequency that is shifted. Apart from $0.15 \lambda$, the ECC is found to be lower than 0.5 . This is because both of the antenna are exciting at the same direction at low frequency with the effect of ground plane modification. The total efficiency is increased and reached the peak at $0.2 \lambda$ width. From $0 \lambda$ to $0.15 \lambda$, the total efficiency is found to be lower than $50 \%$. This is mainly caused by the low radiation efficiency of the antennas.

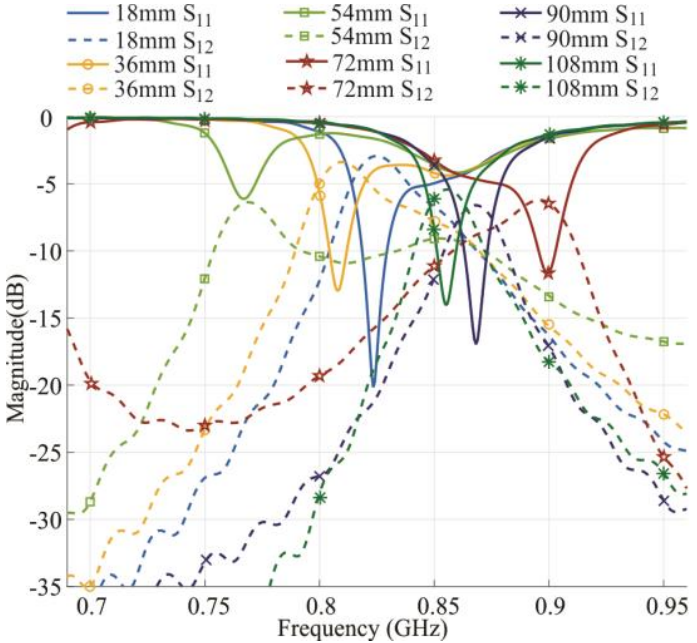

Figure 2. Effect on S-parameters by varying parameter $\mathrm{L}$ of defected ground slot

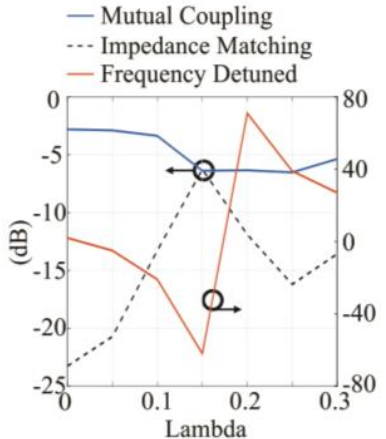

(a)

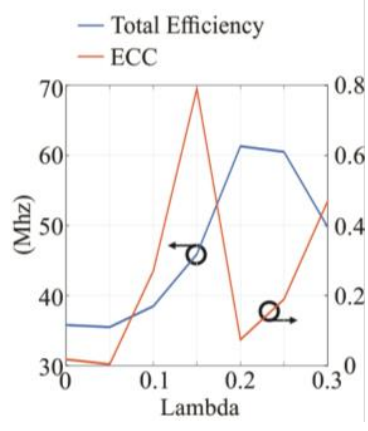

(b)
Figure 3. Performance of (a) S-parameter, (b) total efficiency and ECC for parameter sweep, $\mathrm{L}$

In second part, $\mathrm{W}$ is varied from $0.022 \lambda$ to $0.133 \lambda$ and $\mathrm{L}$ is fixed at $0.25 \lambda$. The direction of $\mathrm{f}_{\mathrm{c}}$ is detuned toward a higher frequency as shown in Figure 4 . The maximum detuned of $f_{c}$ is $331 \mathrm{MHz}$ and the $f_{c}$ is shifted towards a higher frequency at $1.16 \mathrm{GHz}$. Figure 5(a) shows a decrease in the impedance matching of the antennas. The antennas are resonating until it achieves $\mathrm{W}=0.08 \lambda$ at $-6.9 \mathrm{~dB}$. When $\mathrm{W}$ exceed $0.08 \lambda$, the antennas stop operate as the minimum tolerance level of impedance matching is reached. The maximum impedance matching achieved at $-20 \mathrm{~dB}$ and the minimum mutual coupling is at $-6.2 \mathrm{~dB}$. The mutual coupling between the antennas is found to be inversely proportional to W. However, the impedance matching is degraded as W increased. Figure 5(b) shows the total efficiency and ECC for parameter sweep, W. The ECC is gradually increased with the changes in $\mathrm{W}$ and is under 0.5 overall. Total efficiency is at the range of $50 \%$ to $70 \%$. A high mismatch loss at wider $\mathrm{W}$ results in a reduction in the performance of total efficiency.

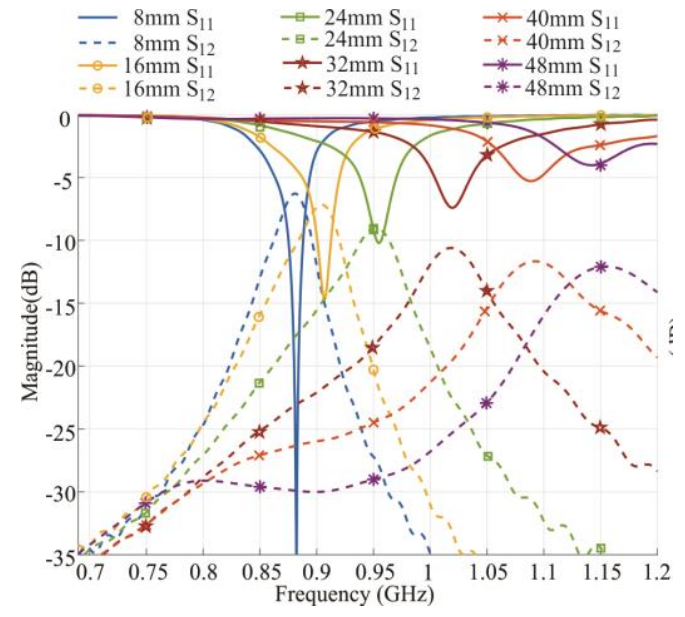

Figure 4. Effect on S-parameters by varying parameter $\mathrm{W}$ of defected ground slot

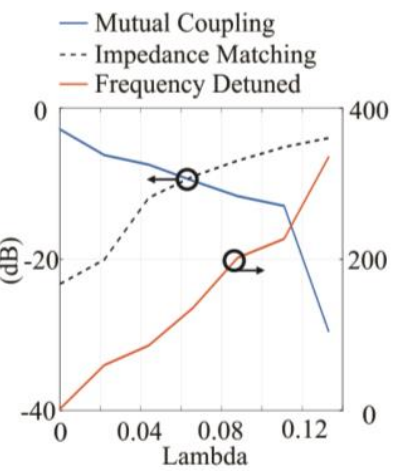

(a)

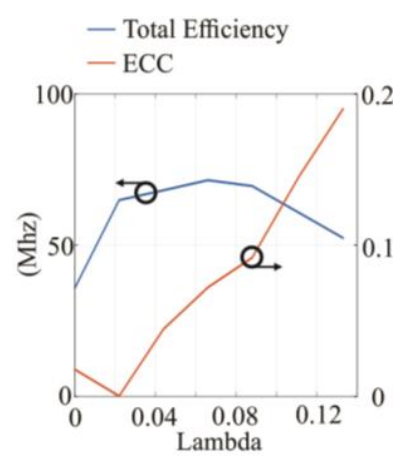

(b)
Figure 5. Performance of (a) S-parameter, (b) total efficiency and ECC for parameter sweep, W 


\section{OPTIMIZATION OF DEFECTED GROUND PLANE}

The optimized dimension of the defected ground plane based on the parameter study aforementioned in Section II is presented in this section. The results of single and two slots on defected ground are discussed in this section.

\subsection{Single slot}

$\mathrm{L}$ and $\mathrm{W}$ of the slot are optimized based on the parameter study in Section II. The modification in ground plane caused a detuned frequency. The dimensions of $\mathrm{L}$ and $\mathrm{W}$ of the antenna are optimized so that the $f_{c}$ is tuned to the desired frequency. Figure 1 shows the dimension of the antenna element with $23 \mathrm{~mm} \mathrm{x}$ $20 \mathrm{~mm}$. The antenna element is optimized by tuning the length of AL. An increase in length of AL shifts the $\mathrm{f}_{\mathrm{c}}$ to a lower frequency and vice versa.

The location of the defected ground slot is remained at the center part at top edge. Dimension of the optimized slot is shown in Figure 6(a). The optimized dimension of $\mathrm{W}$ and $\mathrm{L}$ are $0.0042 \lambda$ and $0.278 \lambda$, respectively. The dimension of L (i.e., $0.278 \lambda$ ) is more than a quarter wavelength at low frequency (i.e., 829 $\mathrm{MHz}$ ). The dimension of AL is tuned to $35 \mathrm{~mm}$ so that the $\mathrm{f}_{\mathrm{c}}$ could be shifted to the operating frequency. The optimized result obtained from simulation based on the parameter study is shown in Figure 6(b). The Sparameter result of optimized single defected ground slot is compared to a full ground antenna. The impedance matching and mutual coupling of the PIFA with full ground plane are $-23 \mathrm{~dB}$ and $-2.85 \mathrm{~dB}$, respectively. The impedance matching and mutual coupling with the implementation of optimized single defected ground slot are $-15 \mathrm{~dB}$ and $-7 \mathrm{~dB}$, respectively. The mutual coupling with the single defected ground slot is able to improve by $-4.15 \mathrm{~dB}$. Impedance matching is reduced by $5 \mathrm{~dB}$ with the compensation of mutual coupling. This is still under the tolerance level of mismatch loss at $-6 \mathrm{~dB}$ [17]. Figure 6(c) shows the radiation pattern of the antenna. The left and right figures in Figure 6(c) denotes the excitation of ports 1 and 2 , respectively. The radiation patterns are obtained at $829 \mathrm{MHz}$. The ECC and total efficiency achieved with single defected ground slot is 0.175 and $62 \%$, respectively.

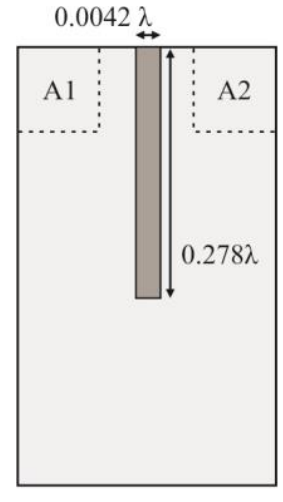

(a)

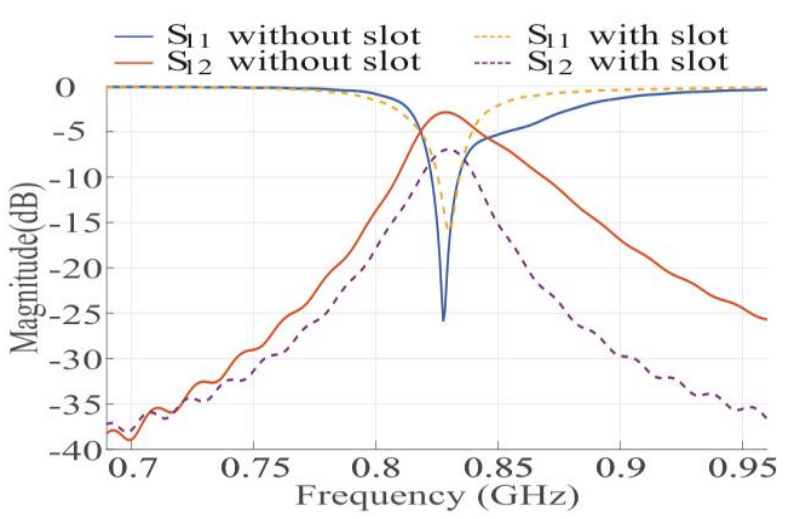

(b)
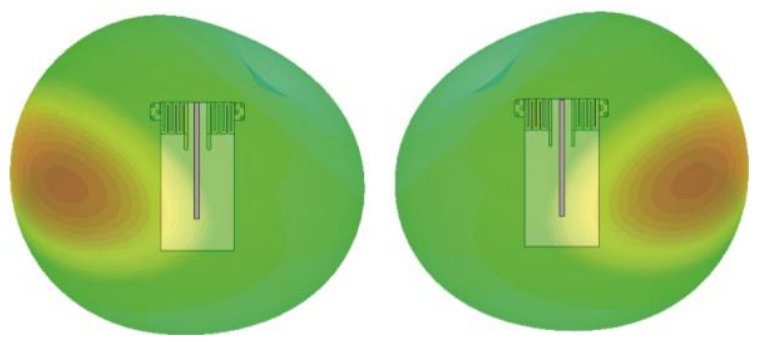

(c)

Figure 6. (a) Dimension of antenna with single defected ground slot, (b) S-parameter comparison with and without defected ground slot, (c) Radiation pattern of each port at $829 \mathrm{MHz}$ 


\subsection{Two slot}

In addition, this study presents the information on multiple defected ground slots with PIFA. Figure 7 (a) shows the defected ground slot. The dimension of the defected ground slot is remained the same: 0.0042 $\lambda \times 0.278 \lambda$. The gap between the slots implemented is $1 \mathrm{~mm}$. The results of the S-parameter with single defected ground slot is compared to two defected ground slots. The results are shown in Figure 7(b). The impedance matching and mutual coupling of two defected ground slots give the same pattern in result with single defected ground slot. The impedance matching and mutual coupling of two defected ground slots are $14.5 \mathrm{~dB}$ and $-7.2 \mathrm{~dB}$, respectively. The difference of impedance matching and mutual coupling with single defected ground slot are $-0.5 \mathrm{~dB}$ and $-0.2 \mathrm{~dB}$. Figure 7 (c) shows the radiation pattern of each port at 829 $\mathrm{MHz}$. The radiation patterns are found to be similar to the single defected ground slot. The ECC and the total efficiency are 0.182 and $56 \%$, respectively. This minor differences are neglectable and is hypothesized to provide the same result.

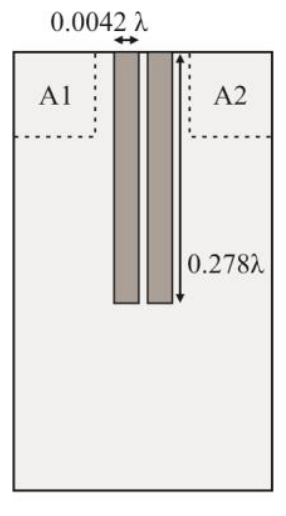

(a)

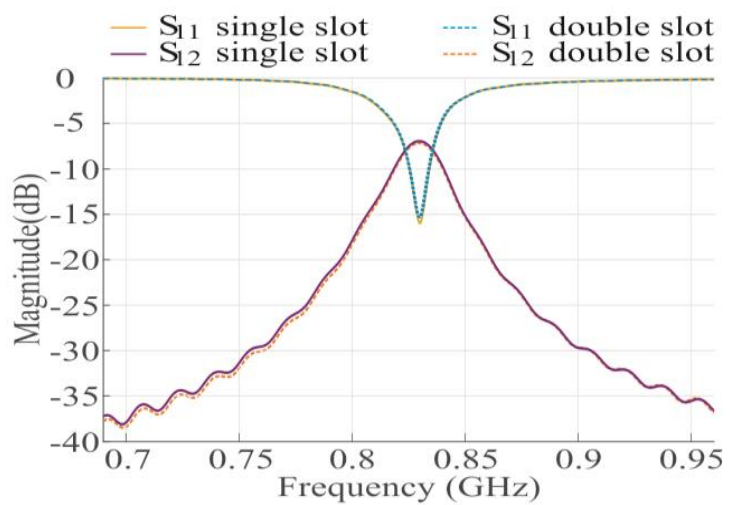

(b)
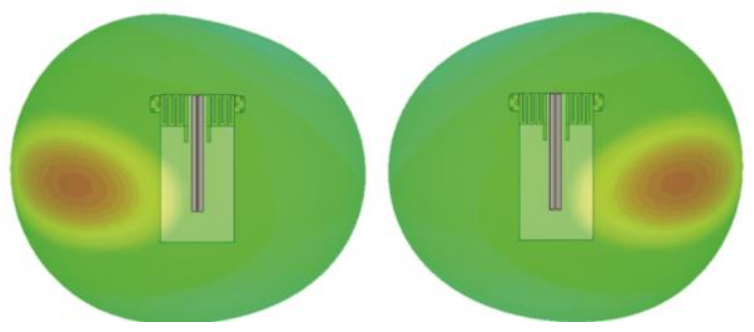

(c)

Figure 7. (a) Dimension of antenna with two defected ground slots, (b) S-parameter comparison of single and two defected ground slots, (c) Radiation pattern of each port at $829 \mathrm{MHz}$

\section{CONCLUSION}

In this paper, a simple defected ground plane with a straight slot is implemented and the studies of the parameters are presented. In the beginning of this paper, the antenna is operating at frequency of 829 $\mathrm{MHz}$ with mutual coupling of $-2.8 \mathrm{~dB}$. The defected ground slot is optimized based on the output of parameters study ( $W$ and $L$ ) and the antenna length, $A L$ to reduce the mutual coupling at the $f_{c}$. The optimized result is implemented with a single defected ground slot of dimension: $0.0042 \lambda \times 0.278 \lambda$. The mutual coupling in this study is further reduced by $4.5 \mathrm{~dB}$ and achieved a minimum value of $-7 \mathrm{~dB}$ with the implementation of defected ground slot. Besides, this study simulated and compared among single and two defected ground slot. Both of the results presented a similar performance which is neglectable. In terms of antenna design, less modification of the ground plane is preferable. This study found that the minimum mutual coupling is at $-7 \mathrm{~dB}$ and the ECC is below 0.5 with a quarter wavelength defected ground slot. Mutual coupling below $-10 \mathrm{~dB}$ at low frequency is difficult to be achieved with rectangular defected ground slot alone without the support of other decoupling techniques. 


\section{ACKNOWLEDGEMENTS}

This research was supported financially by the Malaysian Ministry of Science, Technology and Innovation (MOSTI) under Science fund (Project no: 01-01-015-SF0258)

\section{REFERENCES}

[1] S. Wang and Z. Du, "A Dual-Antenna System for LTE/WWAN/WLAN/WiMAX Smartphone Applications," IEEE Antennas Wirel. Propag. Lett., vol. 14, no. c, pp. 1443-1446, 2015.

[2] C. W. Loon, A. A. Al-Hadi, P. J. Soh and M. F. Jamlos, "Recent developments in multiple antenna mutual coupling compensation techniques for mobile terminals," 2015 IEEE Student Conference on Research and Development (SCOReD), Kuala Lumpur, 2015, pp. 561-564.

[3] M. Pelosi, M. B. Knudsen and G. F. Pedersen, "Mutual coupling in MIMO antennas with transceiver separation," Proceedings of the 5th European Conference on Antennas and Propagation (EUCAP), Rome, 2011, pp. 131-133.

[4] G. P. Kumar and S. S. Babu, "Design of multiband MIMO antenna in compact mobile handsets and SAR distribution," 2015 International Conference on Communications and Signal Processing (ICCSP), Melmaruvathur, 2015, pp. 1501-1505.

[5] H. Li, J. Xiong, Z. Ying and S. He, "High isolation compact four-port MIMO antenna systems with built-in filters as isolation structure," Proceedings of the Fourth European Conference on Antennas and Propagation, Barcelona, 2010, pp. 1-4.

[6] J.-F. Li, Q.-X. Chu, T.-G. Huang, "A compact wideband MIMO antenna with two novel bent slits", IEEE Trans. Antennas Propag., vol. 60, no. 2, pp. 482-489, Feb. 2012.

[7] Zulkifli, F. Y., E. T. Rahardjo, and D. Hartanto, "Mutual coupling reduction using dumbbell defected ground structure for multiband microstrip antenna array," Progress In Electromagnetics Research Letters, Vol. 13, pp. 29-40, 2010.

[8] R. Kumar and R. V. S. R. Krishna, "A dual polarized UWB slot antenna with kite shaped slot for high isolation," International Conference on Microwave, Optical and Communication Engineering (ICMOCE), pp. 261-263, 2015.

[9] H. Huang and J. Wu, "Decoupled Dual-Antenna With Three Slots and a Connecting Line for Mobile Terminals," IEEE Antennas Wirel. Propag. Lett., vol. 14, pp. 1730-1733, 2015.

[10] Z. Li, Z. Du, M. Takahashi, K. Saito, and K. Ito, "Reducing mutual coupling of MIMO antennas with parasitic elements for mobile terminals," IEEE Trans. Antennas Propag., vol. 60, no. 2 part 1, pp. 473-481, 2012.

[11] Q. Sun et al., "Broadband two-element array with hybrid decoupling structures for multimode mobile terminals", IEEE Antennas Wirel. Propag. Lett., vol. 14, pp. 1431-1434, 2015.

[12] G. Li, H. Zhai, Z. Ma, C. Liang, S. Member, and R. Yu, "Isolation- Improved Dual-Band MIMO Antenna," IEEE Antennas Wirel. Propag. Lett., vol. 13, pp. 1128-1131, 2014.

[13] P. S. Kumar and B. C. Mohan, "Design of a compact two element MIMO antenna with improved bandwidth and isolation," 2015 International Conference on Microwave, Optical and Communication Engineering (ICMOCE), Bhubaneswar, 2015, pp. 389-392.

[14] S. N. Venkatasubramanian, L. Li, A. Lehtovuori, C. Icheln, K. Haneda, "Impact of using resistive elements for wideband isolation improvement", IEEE Trans. Antennas Propag., vol. 65, no. 1, pp. 52-62, Jan. 2017.

[15] S. C. Chen, Y. S. Wang, S. J. Chung, "A decoupling technique for increasing the port isolation between two strongly coupled antennas", IEEE Trans. Antennas Propagat., vol. 56, pp. 3650-3658, Dec. 2008.

[16] W. L. Cheor, A. A. Al-Hadi, P. J. Soh, and M. F. Jamlos, "Impact of Antenna Types and Locations on Small Multielement Antenna Performance for Low-band LTE Mobile Terminals" IEEE Asia-Pacific Conference on Applied Electromagnetics (APACE), pp. 310-314, 2016.

[17] S. Zhang, K. Zhao, Z. Ying, and S. He, "Investigation of Diagonal Antenna - Chassis Mode in Mobile Terminal LTE MIMO Antennas for Bandwidth Enhancement," IEEE Antennas Propag. Mag., vol. 57, no. 2, pp. 217-228, 2015 .

\section{BIOGRAPHIES OF AUTHORS}

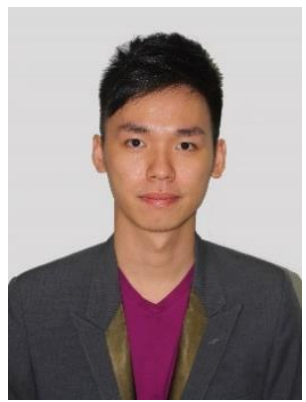

Wai Loon Cheor was born on March 1990 in Perak, Malaysia. He received B.Eng. degree in Communication Engineering from Universiti Malaysia Perlis (UniMAP), Malaysia in 2014. He is currently working toward his Ph.D. degree in Communication Engineering at the same university. His research interests are multi-element antennas and mobile terminal antennas. 


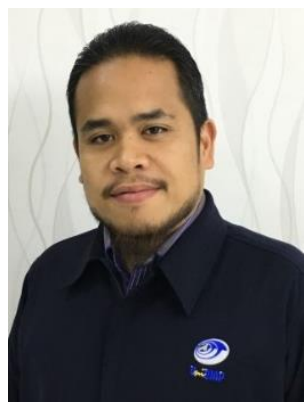

Azremi Abdullah Al-Hadi (S'13 M'13 SM'14) was born on August 26 in Michigan, United States of America. He received the Master of Science degree in communication engineering from Birmingham University, United Kingdom in 2004 and the Doctor of Science in Technology degree from Aalto University, Finland in 2013. His current research interests include design and performance evaluation of multi-element antennas, mobile terminal antennas and their user interactions, and wireless propagation. He is currently working as an Associate Professor and holds position as Dean of the School of Computer and Communication Engineering, Universiti Malaysia Perlis (UniMAP). He has been with the school since 2002. He is active in volunteer work with IEEE Malaysia Section, acting as the Senior Member of IEEE, Vice Chair of the IEEE Antenna Propagation/Microwave Theory techniques / Electromagnetic Compatibility (AP/MTT/EMC) Malaysia Chapter and Counselor for the IEEE UniMAP Student Branch. He is the Chartered Engineer of the Institution of Engineering and Technology (IET), UK, the member of the Board of Engineers Malaysia (BEM), Malaysia and graduate technologist of the Malaysia Board of Technologist (MBOT), Malaysia. Dr. Azremi was the recipient of the Best Student Paper Award presented at the $5^{\text {th }}$ Loughborough Antennas and Propagation Conference (LAPC 2009) and the CST University Publication Award in 2011.

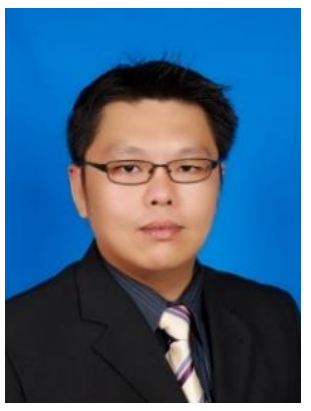

Ping Jack Soh (S '10, M '14, SM '15) was born in Sabah, Malaysia. He received the B.Eng. and M.Eng. degrees in Electrical Engineering (Telecommunication) from Universiti Teknologi Malaysia (UTM) in 2002 and 2006, respectively, and the PhD degree in Electrical Engineering from KU Leuven, Belgium in 2013. He is currently an Associate Professor at the School of Computer and Communication Engineering (SCCE), Universiti Malaysia Perlis (UniMAP), and also a Research Affiliate at KU Leuven, Belgium. Dr. Soh was the recipient of the IEEE Antennas and Propagation Society (AP-S) Doctoral Research Award in 2012, the IEEE Microwave Theory and Techniques Society (MTT-S) Graduate Fellowship for Medical Applications in 2013 and the International Union of Radio Science (URSI) Young Scientist Award in 2015. He is a Chartered Engineer registered with the UK Engineering Council; a Senior Member of the IEEE, a Member of the IET, ACES and URSI; and Graduate Members of the Board of Engineers Malaysia (BEM), the Institution of Engineers Malaysia (IEM) and the Malaysia Board of Technologist (MBOT).

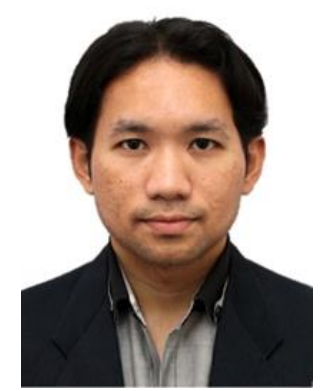

Mohd Faizal Jamlos received Ph.D. in 2010 from Universiti Teknologi Malaysia, Johor, Malaysia and M.Sc. in 2008 from University of Adelaide, South Australia, Australia. He is currently Associate Professor at Advanced Communication Engineering Centre (ACE), School of Computer and Communication Engineering, Universiti Malaysia Perlis. He has (co-)authored some 220 scientific publications in peer-reviewed journals and conferences. His research interest are wireless embedded system, remote sensing, on-platform antennas and microwave circuitry. $\mathrm{He}$ is a practice professional Engineer of Board of Engineers Malaysia (BEM), Senior Member of IEEE, a National Medical Researcher (NMRR) and Corporate Member of Institute Engineers Malaysia (MIEM). 